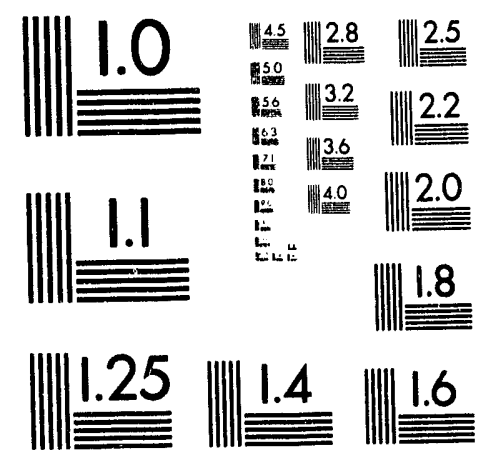



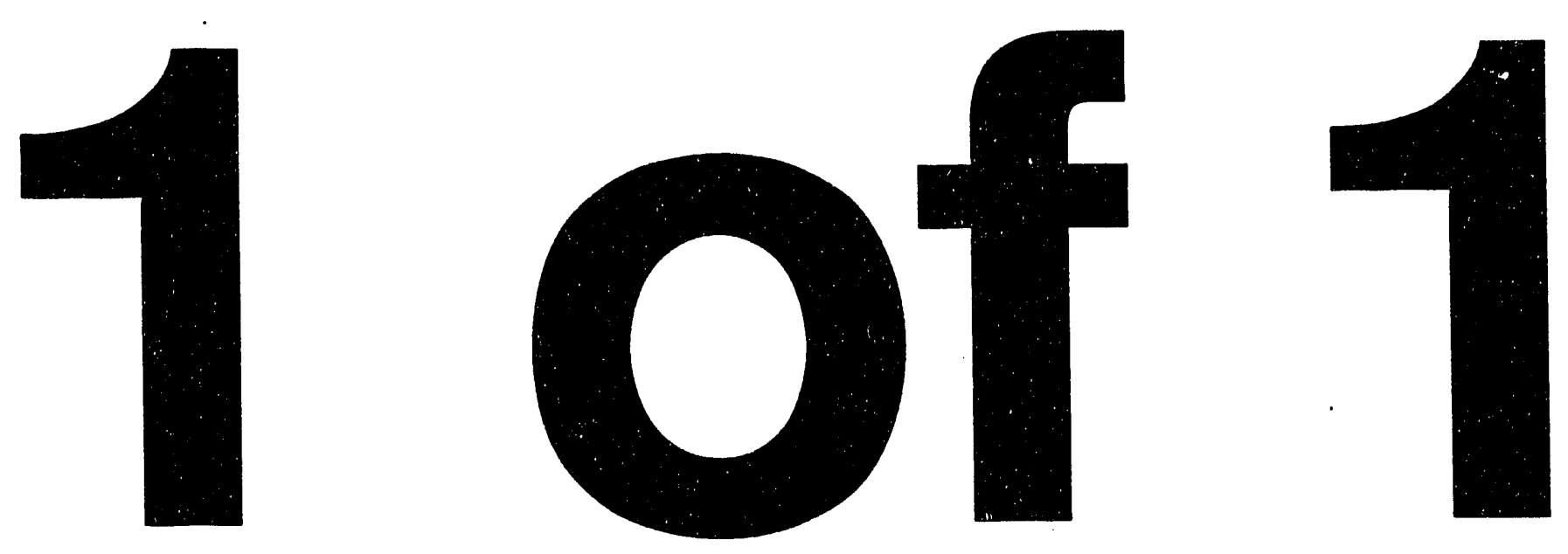


\title{
Electron Quantum Yields from a Barium Photocathode Illuminated with Polarized Light*
}

\author{
M. E. Conde, S. Chattopadhyay, K.-J. Kim, \\ S.-I. Kwon ${ }^{\dagger}$, K.-N. Leung, and A. T. Young \\ Lawrence Berkeley Laboratory \\ University of California, Berkeley, CA 94720 USA
}

Abstract

Photoemission measurements with a barium photocathode and a nitrogen laser are reported. The cathode is prepared by evaporating barium onto a copper disc. Radiation from a nitrogen laser ( $337 \mathrm{~nm}, 10 \mathrm{~ns})$ is polarized and strikes the cathode surface at variable angles. An electron quantum yield as high as $1 \times 10^{-3}$ is observed. The dependence of the quantum yield on the beam polarization and angle of incidence is investigated. The results indicate that higher quantum yields are achieved when the laser beam is incident at an angle of $-55^{\circ}$ and is polarized perpendicular to the plane of incidence.

\section{INTRODUCTION}

The laser driven RF photocathode [1] holds great promise as a source of tightly bunched high-brightness electron beams. This is important for many accelerator applications, as well as for the generation of shortwave electromagnetic radiation. It is therefore important to improve and optimize the photocathode efficiency.

It has been shown [2] that barium is an attractive candidate for use as a photocathode material. It has a low work function $(-2.5 \mathrm{eV})$ and provides relatively high electron quantum yields. This paper reports work on the optimization of the performance of barium photocathodes. Studies are conducted on the dependence of the quantum yield on laser beam incident angle and polarization. The results shown bere may be relevant to other photocathode materials as well.

\section{EXPERIMENTAL SETUP}

A $22 \mathrm{~cm}$ diameter stainless-steel vacuum chamber pumped by a cryosorption pump houses the photocathode. The typical base pressure in the chamber is $2 \times 10^{-7}$ Torr. The cathode consists of a solid copper disc (20 $\mathrm{mm}$ in diameter) onto which a thin layer of barium is vapor deposited. The anode, a graphite disc (15 $\mathrm{mm}$ in diameter), is located directly in front of the cathode. They are mounted on a stainless-steel shaft by means of small ceramic rods and separated by a distance of 40 $\mathrm{mm}$ (Fig. 1). The rotation of the shaft allows for the variation of the laser incident angle on the cathode surface, while keeping the cathode-anode distance fixed.

The photocathode is prepared by evaporating barium from commercially available getter wires (SAES). A moveable support, holding twenty $1.6 \mathrm{~cm}$-long segments of barium

* This work was supported by the Director, Office of Energy Research, Office of Fusion Energy, Development and Technology Division, of the U.S. Department of Energy under Contract No. DEAC03-76SF00098.

$\dagger$ Permanent address: Department of Physics, Kyonggi University, Suwon, Korea. getter wire, is placed directly in front of the cathode surface. The passage of electric current through the wires (approximately 5 Amperes per segment) raises their temperature and causes the barium to evaporate. Some of the barium vapor adheres to the cathode surface, forming a barium layer that is a few microns thick. The getter assembly is then moved away from the cathode. The whole process of barium deposition is performed in vacuum, and the pressure is kept in the $10^{-7}$ Torr range.

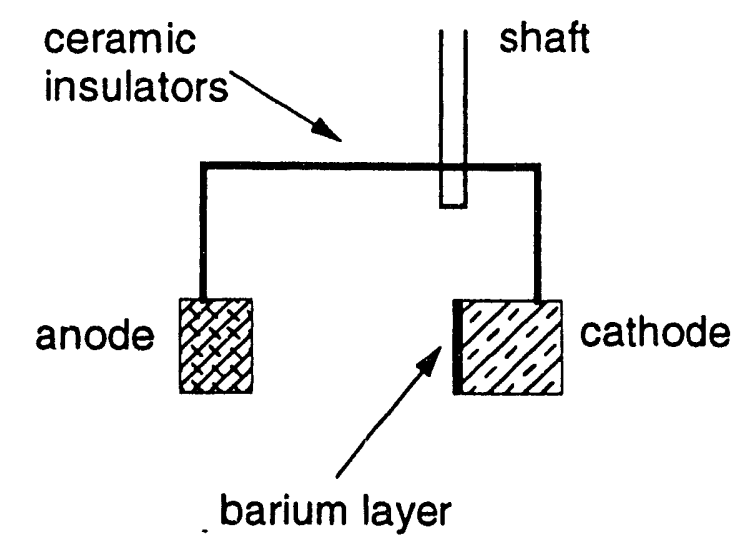

Figure 1. Schematic of the cathode and anode.

A schematic of the experimental setup is shown in Fig. 2. Nitrogen laser radiation at $337 \mathrm{~nm}$ with a pulse width of approximately $10 \mathrm{~ns}$ and a repetition rate of $22 \mathrm{~Hz}$ is used to excite the photocathode. An iris limits the diameter of the beam to approximately $4 \mathrm{~mm}$. The laser beam is directed through a spatial filter to improve the beam quality and polarized by a calcite Glan prism. The beam power is then attenuated to $0.05-1 \mu \mathrm{J} /$ pulse and enters the vacuum chamber through a quartz window. The photoelectrons are collected by the anode, which can be biased to a maximum potential of 6 $\mathrm{kV}$.

A photodiode monitors the laser power by using the small fraction of the beam that is reflected at the quartz window. It is important to note that this reflection occurs at nearnormal incidence, making the reflection coefficient insensitive to the polarization state of the beam. This reflected beam is subsequently deflected to the photodiode by a prism. (This process is also insensitive to the beam polarization.)

\section{MEASUREMENTS}

The time-averaged photocurrent is measured by a picoammeter connected to the cathode. The quantum yield is then calculated as the ratio between the number of emitted electrons and the number of incident photons. A calibrated thermopile detector (Scientech, model S-3610) is used to mea- 


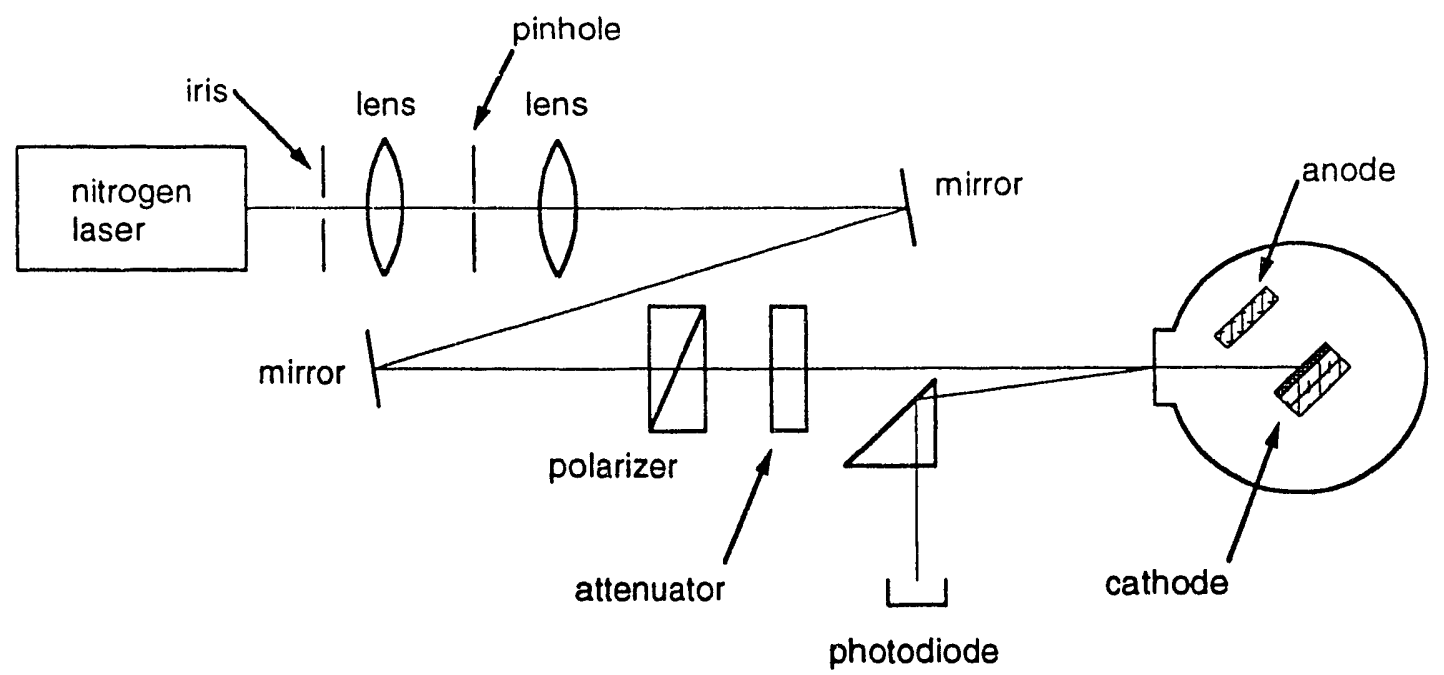

Figure 2. Schematic of the experimental setup.

sure the time-averaged laser power, thereby determining the number of photons.

Figure 3 presents the measured quaritum yield as a function of anode bias voltage and barium layer age. The graph shows that the voltage applied to the collecting anode has to be sufficiently high to overcome the space-charge limit at the cathode. For high enough voltages the measured quantum yield is independent of the anode voltage, indicating that all the emitted electrons are collected. The figure also illustrates the decrease of the quantum yield as a function of the cathode age, which is presumably a consequence of the contamination of the barium surface.

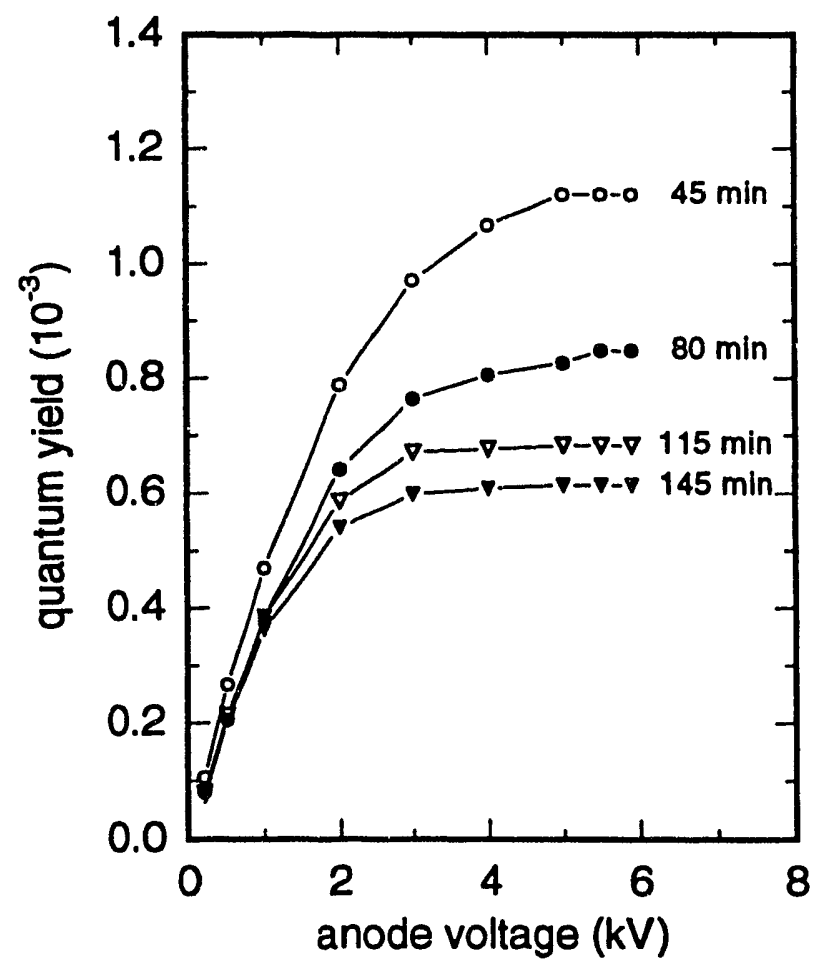

Figure 3. Electron quantum yield as a function of anode voltage and barium layer age.
Absolute values of the laser beam energy are measured with the above mentioned thermopile detector. The precision of these power measurements is $-20 \%$ at these low average power levels $(<0.5 \mathrm{~mW})$. More precise (although only relative) power measurements are obtained with a photodiode (see Fig. 2). In the next paragraphs we present the quantum yield dependence on the polarization and angle of incidence of the laser beam. In both cases the laser power is measured with the photodiode, therefore the quantum yield values are just relative measurements.

Figure 4a shows the relative quantum yield as a function of laser beam polarization (for a fixed angle of incidence). The quantum yield reaches a maximum value when the polarization state is such that the component of the electric field normal to the cathode surface is maximized (cf. Fig. 4b). Conversely, the quantum yield has a minimum value when the electric field in the laser beam is parallel to the cathode surface.

Figure 5 illustrates the dependence of the quantum yield on the laser beam incident angle. Two curves are shown, corresponding to two polarization angles of the beam (perpendicular to each other). The difference between the quantum yield of each polarization becomes larger as the incident angle is increased. The maximum quantum yield is reached at an incident angle of $-55^{\circ}$, with the beam polarized perpendicular to the plane of incidence. Small angles of incidence cannot be explored because the anode would block the laser beam. Measurements with very large angles are also difficult; a fraction of the beam can easily "miss" the cathode surface.

\section{DISCUSSION}

In these experiments electron quantum yields of $10^{-3}$ have been measured with a barium photocathode at a wavelength of $337 \mathrm{~nm}$. These values are comparable to results in similar experiments employing barium cathodes and at a number of excimer laser wavelengths [2]. Thus, it has been demonstrated that barium is a very attractive candidate for use as a photocathode material. Barium presents quantum yields much higher than the values obtained with bare copper, with vacuum requirements that are only slightly more stringent 
$\left(-10^{-7}\right.$ Torr). Cesiated semiconductors [3] can achieve higher quantum yields, but ultra-high vacuum is required for their operation $\left(<10^{-9}\right.$ Torr $)$.
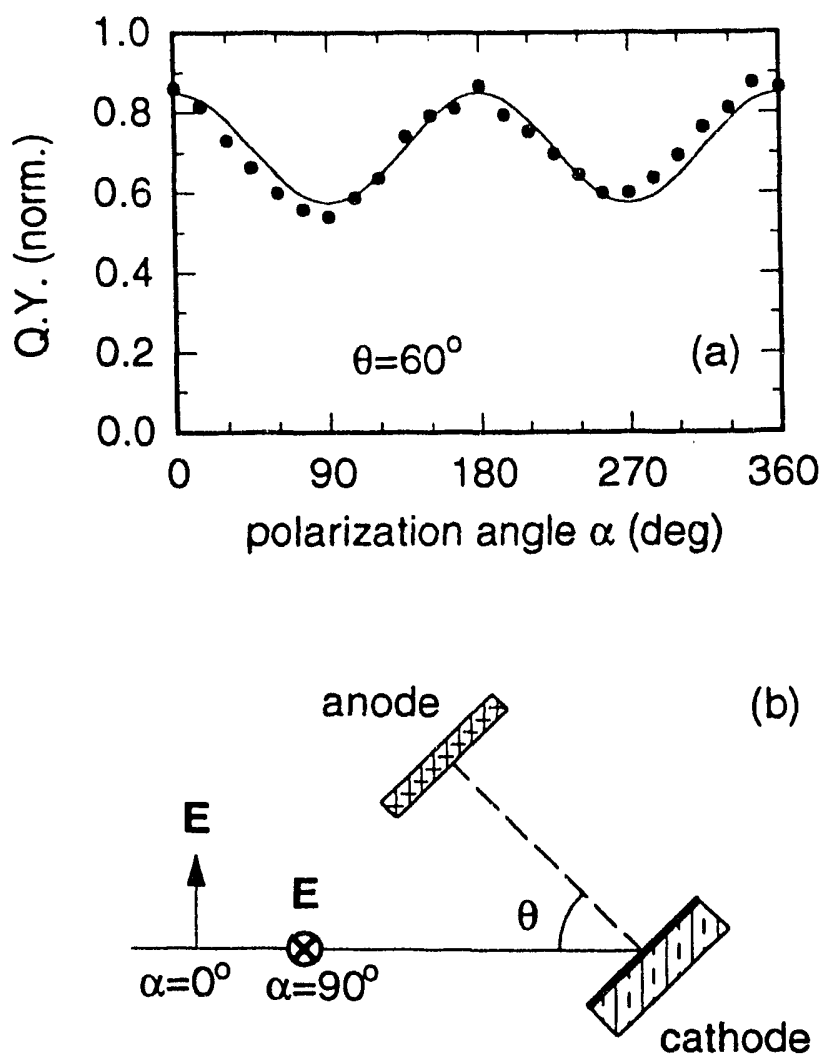

Figure 4. Effect of the laser polarization on the quantum yield: (a) normalized quantum yield as a function of polarization angle; (b) diagram indicating the angles of polarization $(\alpha)$ and incidence $(\theta)$.

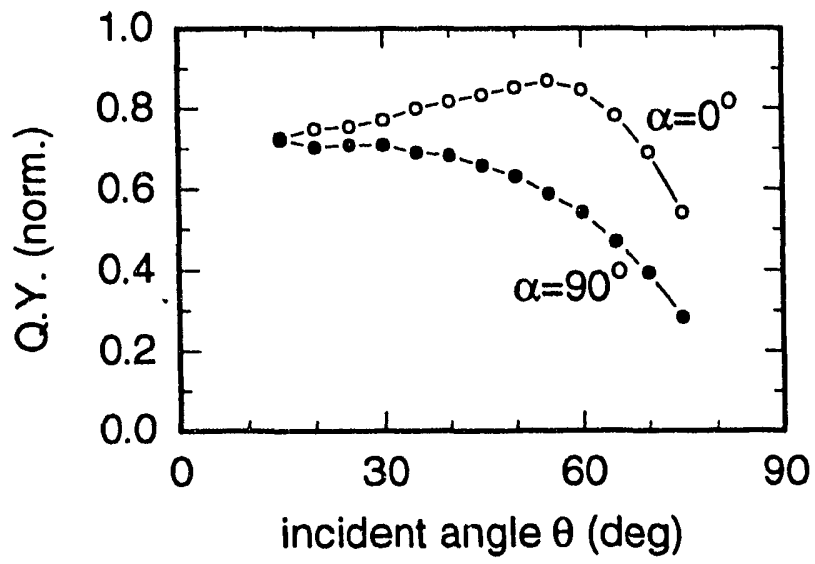

Figure 5. Normalized quantum yield as a function of angle of incidence.
An enhancement of the photoemission has been observed when the laser light is polarized such that the electric field of the laser beam has a component normal to the cathode surface. This effect is small at near-normal laser incidence, and is more pronounced for larger incident angles.

The effect of the beam polarization on the quantum yield must be, in part, due to changes of the reflection coefricient of the barium as a function of the angle of incidence and the polarization of the light. Thus, a smaller reflection coefficient allows a larger fraction of the incident photons to interact with the barium layer, yielding a larger number of photoelectrons. We are presently investigating this topic. Preliminary calculations of the reflection coefficient are consistent with the measured curves. These estimates are limited by the uncertainty in the values of the optical constants of barium, and also by the unknown effect of the roughness of the actual cathode surface.

We have also observed these polarization effects with laser power levels that differ by an order of magnicude. This suggests that the effect cannot be attributed to field-assisted photoemission in an obvious way.

\section{ACKNOWLEDGEMENTS}

We thank Wim Leemans for advice on the optics and for lending us the photodiode. We are aiso grateful to John Edighoffer for helpful discussions.

\section{REFERENCES}

[1] R. L. Sheffield, "Photocathode RF Guns," AIP Conference Proceedings \#184, Physics of Particle Accelerators, edited by M. Month and M. Dienes, AIP 1989, p. 1500.

[2] M. D. Vanloy, A. T. Young, and K.-N. Leung, "Measurements of Barium Photocathode Quantum Yields at Four Excimer Laser Wavelengths," (submitted to Appl. Phys. Lett.).

[3] P. E. Oettinger, R. E. Shefer, D. L. Birx, and M. C. Green, "Photoelectron Sources: Selection and Analysis," Nucl. Instrum. Methods A272, 264 (1988). 

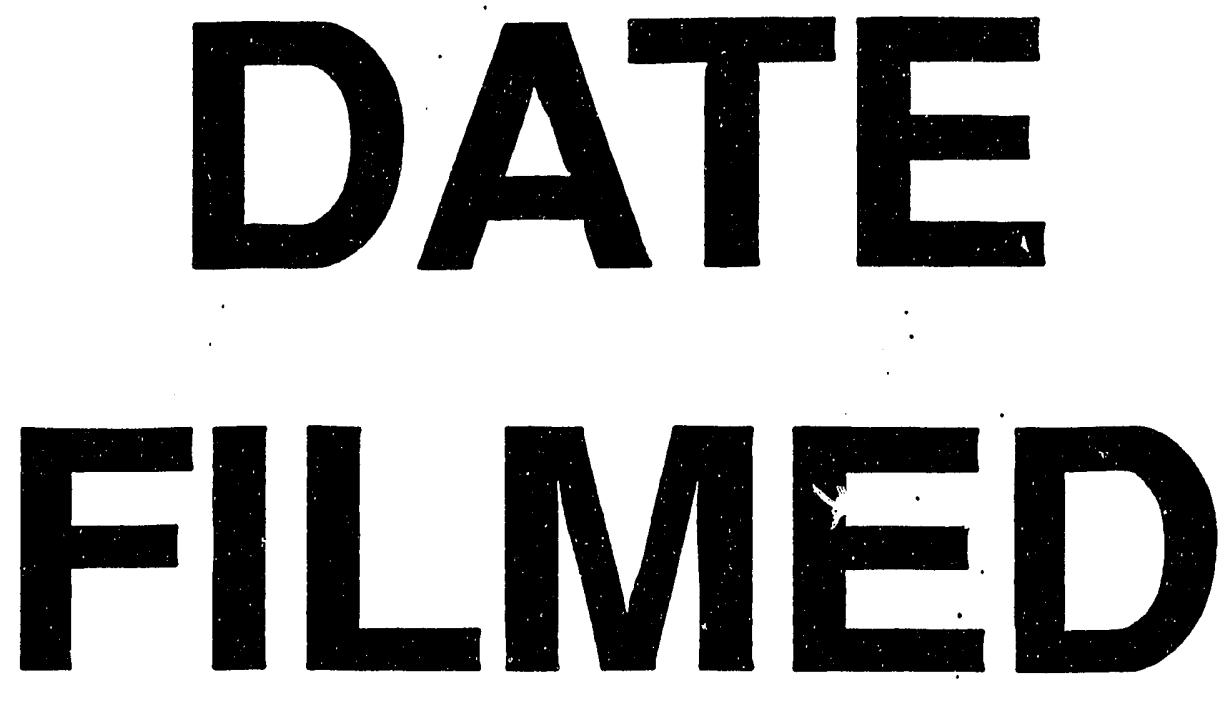

$10 / 20 / 93$
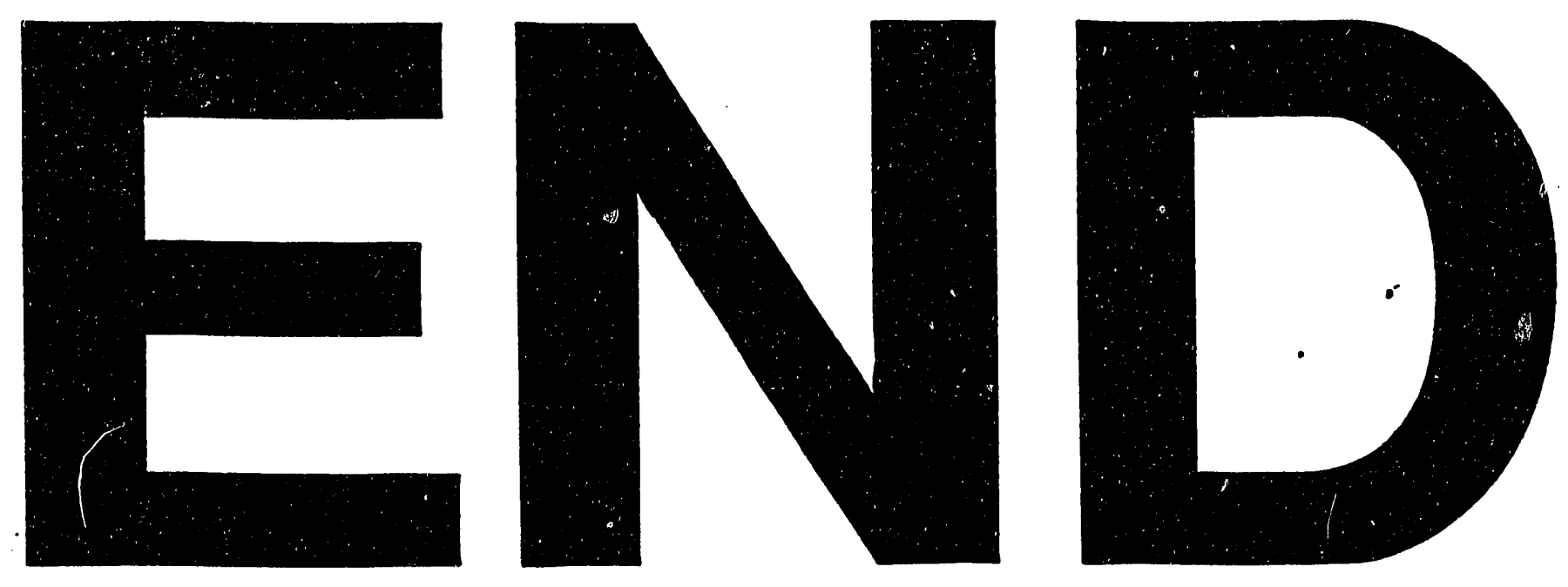
